\section{O Sistema de Informação da Atenção à Saúde Indígena (SIASI): criação, estrutura e funcionamento}

\author{
The Health Information System for Indigenous \\ Peoples in Brazil (SIASI): design, structure, \\ and functioning
}

\author{
${ }^{1}$ Coordenação Regional \\ de Mato Grosso, Fundação \\ Nacional de Saúde, Cuiabá, \\ Brasil. \\ 2 Instituto de Saúde Coletiva, \\ Universidade Federal de \\ Mato Grosso, Cuiabá, Brasil. \\ 3 Escola Nacional de Saúde \\ Pública Sergio Arouca, \\ Fundação Oswaldo Cruz, \\ Rio de Janeiro, Brasil. \\ 4 Departamento de \\ Antropologia, Museu \\ Nacional, Rio de Janeiro, \\ Brasil. \\ Correspondência \\ M. C. Sousa \\ Coordenação Regional de \\ Mato Grosso, Fundação \\ Nacional de Saúde. \\ Av. Bosque da Saúde 355, \\ Cuiabá, MT 78050-070 \\ Brasil. \\ conceicaosousa@terra.com.br
}

\begin{abstract}
The Brazilian Health Information System for Indigenous Peoples (SIASI) was created in 1999 as part of the Sub-System for Indigenous Peoples' Health Care. The aim was to improve availability and access to crucial health information for planning, implementation, and evaluation. The current study analyzes the SIASI, focusing on its creation, implementation, functioning, potential, and limitations. Documents were surveyed in the Indigenous Peoples' Health Department of the National Health Foundation, followed by comparative analyses with other health information systems. The SIASI is organized in eight modules, three of which were in operation at the time of the study. The system's main limitations involve data collection instruments, human resources training, lack of interface with other national health information systems, difficult access to the data, and non-utilization of the data to plan subsequent health activities. The potentialities include the system's simplicity, comprehensiveness, and possibilities for disaggregating data. The study highlights the importance of a specific health information system for indigenous peoples, due to their socio-cultural and demographic specificities.
\end{abstract}

Information Systems; Health Policy; South American Indians
Maria da Conceição de Sousa 1

João Henrique G. Scatena 2

Ricardo Ventura Santos 3,4

\section{Introdução}

Como é amplamente reconhecido, a informação é um elemento fundamental nas análises de situações de saúde, seja em nível local, nacional ou internacional. Não somente oferece subsídios para o planejamento e a organização dos serviços de saúde, como é de importância nas etapas de acompanhamento e avaliação 1,2,3.

No tocante aos povos indígenas no Brasil, Coimbra Jr. \& Santos ${ }^{4}$ apontam que a coleta e análise das informações demográficas e epidemiológicas são ainda bastante deficientes. Argumentam que é premente o estabelecimento de um sistema de estatísticas contínuas e confiáveis, preferencialmente integrado aos sistemas nacionais de informação em saúde. Para esses autores, evidenciar os contrastes e as desigualdades da situação de saúde dos indígenas em relação a outros segmentos da sociedade nacional, em particular a partir das informações em saúde, é um passo importante na busca da eqüidade. No presente, as dificuldades em se ter um quadro fidedigno do perfil demográfico e epidemiológico da população indígena estão relacionadas à pouca confiabilidade dos dados existentes, o que limita a realização de análises mais abrangentes $5,6,7,8$.

Em 2000, como parte da Política Nacional de Atenção à Saúde dos Povos Indígenas 9 , foi criado o Sistema de Informação da Atenção à Saúde Indígena (SIASI). Esse sistema visa à coleta, ao 
processamento e à análise de informações para o acompanhamento da saúde das comunidades indígenas, abrangendo óbitos, nascimentos, morbidade, imunização, produção de serviços, recursos humanos e infra-estrutura. Anteriormente ao SIASI, até 1999, as informações de saúde dos povos indígenas eram gerenciadas pela Fundação Nacional do Índio (FUNAI). Em geral, dados vitais, particularmente nascimentos e óbitos, eram coletados nas aldeias sem que fossem consolidados, analisados ou divulgados satisfatoriamente $4,6,10$.

O presente artigo tem como objetivo proceder a uma análise do SIASI, caracterizando sua criação, estrutura e funcionamento no país. Além da comparação com os demais sistemas de informação em saúde existentes, enfocam-se também as possibilidades do sistema para a vigilância e para a geração de informações, tendo em vista sua utilidade na definição de políticas e programas de saúde e no processo de avaliação dos serviços, voltados para os povos indígenas.

\section{Metodologia}

Foi conduzido um estudo descritivo, de abordagem qualitativa, acerca do processo de concepção e implantação do SIASI, tendo como referência o arcabouço jurídico institucional definido pelo Sistema Único de Saúde (SUS), pelo Subsistema de Atenção à Saúde Indígena e pelas recomendações emanadas das conferências nacionais de saúde indígena e documentos normalizadores do próprio SIASI.

Para a caracterização do SIASI e de seu processo de concepção e implantação, foi realizado um amplo levantamento documental, com foco em documentos produzidos pelo nível central da Fundação Nacional de Saúde (FUNASA), em particular junto ao Departamento de Saúde Indígena (DESAI). Procedeu-se uma análise dos documentos de criação do sistema, identificando, entre outros aspectos: origens e lógica do sistema, instrumentos de coleta, variáveis incluídas, indicadores propostos, fluxo das informações, distintas versões do sistema, módulos em funcionamento e desenvolvimento, além das dificuldades operacionais percebidas pelo nível central.

Foi também conduzida análise comparativa das variáveis presentes no SIASI com aquelas coletadas rotineiramente por outros sistemas de informação em saúde existentes no país, em particular pelo Sistema de Informações sobre Mortalidade (SIM) e pelo Sistema de Informação sobre Nascidos Vivos (SINASC).

O projeto que originou esse trabalho foi aprovado pelo Comitê de Ética em Pesquisa do Hospi- tal Júlio Muller, da Universidade Federal de Mato Grosso (protocolo no. 171/2004).

\section{As origens do SIASI}

Em 1999, a responsabilidade pela assistência à saúde dos povos indígenas, até então sob jurisdição da FUNAI, passou para o Ministério da Saúde, por meio da FUNASA. As diretrizes dessa mudança foram definidas na Lei no. 9.836/99 11, que instituiu o Subsistema de Atenção à Saúde Indígena no âmbito do SUS.

Na 1ạ Conferência Nacional de Proteção à Saúde do Índio, realizada em 1986, foram definidos os pressupostos do subsistema. Na ocasião, foi proposto que o gerenciamento das ações e serviços de atenção à saúde indígena deveria ser de responsabilidade do Ministério da Saúde, de modo a integrar o subsistema ao SUS 12. A 2a Conferência Nacional de Saúde para os Povos Indígenas, realizada em 1993, aprimorou a proposta de distritalização sanitária, recomendando a manutenção do Ministério da Saúde como responsável pela atenção sanitária aos povos indígenas e a extensão dos princípios de universalidade, eqüidade, acessibilidade e controle social, oriundos do SUS, para os povos indígenas a serem assistidos 5 .

O modelo de atenção à saúde dos povos indígenas tem como base o Distrito Sanitário Especial Indígena (DSEI), que se constitui como um modelo diferenciado de organização de serviços, voltado para a proteção, promoção e recuperação da saúde, caracterizando-se como um sistema local de saúde 9. Em 1999, foram criados 34 DSEI, distribuídos em todo o território brasileiro. Esses têm uma rede de serviços de atenção básica instalada dentro das terras indígenas, que deve ser integrada, hierarquizada e articulada com a rede do SUS (Figura 1). Nas áreas indígenas, a atenção básica se dá por meio das equipes multidisciplinares, compostas por médicos, enfermeiros, odontólogos, auxiliares de enfermagem e agentes indígenas de saúde e de saneamento.

A necessidade de desenvolver um sistema de informação específico para os povos indígenas foi apontada na 1ạ Conferência Nacional de Proteção à Saúde do Índio, realizada em 1986. No relatório final, foi preconizada a criação de um sistema que fosse capaz de coletar e processar regularmente os dados necessários para uma análise epidemiológica e que permitisse retratar a dinâmica populacional, levando em conta as particularidades socioculturais das centenas de etnias indígenas existentes no país. Além disso, foi preconizada a garantia de que as informações epidemiológicas fossem repassadas às lideran- 


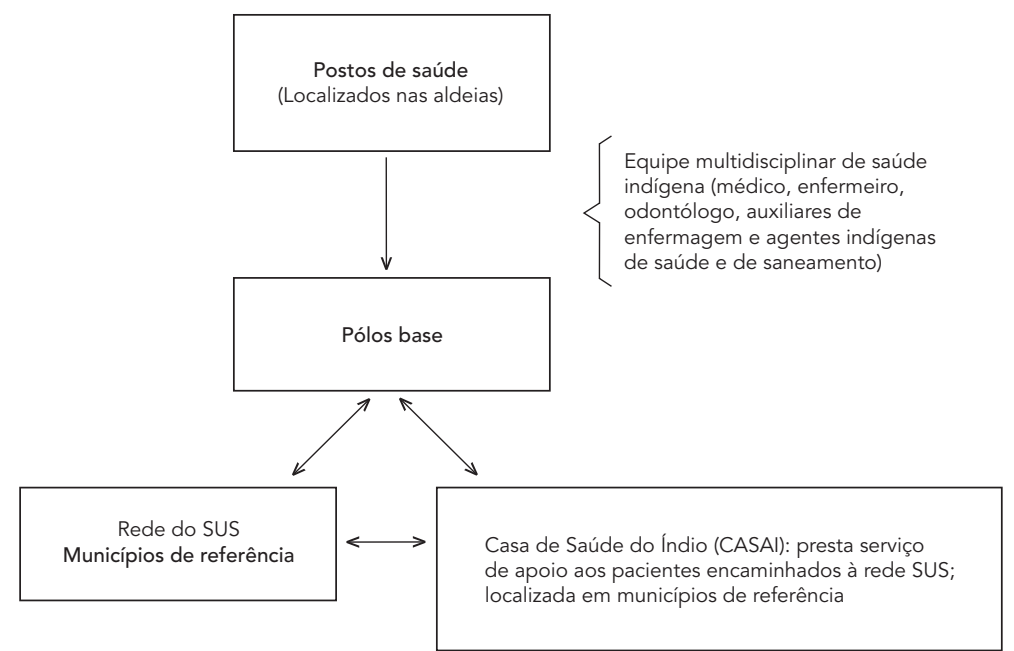

Fonte: Portaria no. 7023

ças indígenas e às autoridades sanitárias $12 . \mathrm{Na}$ conferência seguinte, em 1993, a questão não foi discutida. Na 3a Conferência, realizada em 2001, com o SIASI já criado e em fase de implantação, foi enfatizada a necessidade de intercomunicabilidade com os demais sistemas de informação do Ministério da Saúde e com as bases de dados da FUNAI 13.

Segundo o documento referente à Política Nacional de Atenção à Saúde dos Povos Indígenas 9 (p. 17), “os aspectos a serem acompanhados $e$ avaliados [pelo SIASI] incluirão a estrutura, o processo e os resultados da atenção à saúde dos povos indígenas. O SIASI deverá subsidiar os órgãos gestores e de controle social quanto à indispensável compatibilidade entre o diagnóstico situacional dos problemas de saúde identificados $e$ as prioridades estabelecidas nos níveis técnico, social e político, visando à coerência entre ações planejadas e efetivamente executadas".

O SIASI foi concebido em 1999 e sua implantação iniciada simultaneamente em todos os 34 DSEI em 2000, sendo adotadas as seguintes estratégias: (i) reunião nacional com os chefes dos DSEI, que ocorreu em 2000. Na ocasião, a ênfase das discussões centrou-se primordialmente em questões referentes à informatização do sistema (por exemplo, as possibilidades de relatórios e vantagens para os gestores), com reduzido foco na operacionalização do mesmo; (ii) treina- mentos para as equipes dos DSEI, em geral nas próprias sedes, a partir de dezembro de 2000 . Tal como nas reuniões nacionais, a ênfase era para a dimensão de informática do sistema.

De 2000 a 2002, os DSEI, de forma diferenciada, a depender das condições locais, alimentaram o SIASI na sua modalidade on-line (SIASI Web). Em algumas situações, a alimentação foi diretamente assegurada pelo DESAI, em Brasília. A partir do início de 2002, quando se tornaram cada vez mais evidentes as dificuldades operacionais relacionadas à alimentação on-line dos dados produzidos nos DSEI, houve uma mudança de estratégia do nível central, com a criação do chamado SIASI local, como veremos em detalhes mais adiante. Em meados de 2002, para viabilizar essa nova estratégia, o DESAI iniciou uma série de treinamentos em diversas localidades do país, com a realização de quatro oficinas (em Belém, Pará; Canela, Rio Grande do Sul; Fortaleza, Ceará; e Manaus, Amazonas). Em 2004, foi realizado um outro treinamento nacional (em Belém), dividido em duas fases: uma voltada para treinar profissionais para suporte operacional (instalação e funcionamento do sistema) e outra para a utilização do módulo de imunização, além dos outros módulos já instalados (demográfico e morbidade). 


\section{A lógica de funcionamento do SIASI}

O SIASI foi concebido para funcionar em módulos, assim estruturados: demográfico, morbidade, imunização, saúde bucal, nutrição, acompanhamento à gestação, recursos humanos, infraestrutura e saneamento. Atualmente, apenas os módulos demográfico, de morbidade e de imunização estão implantados (Tabela 1). Houve o desenvolvimento de um outro módulo, o de saneamento, que veio a se constituir em um sistema próprio (SISABI ou Sistema de Informação sobre Saneamento Básico em Áreas Indígenas).

Os diversos módulos possibilitariam a geração de relatórios específicos (indicadores demográficos e de estatísticas vitais, de morbidade, de produtividade, de organização, entre outros). Portanto, teoricamente, o sistema permitiria a avaliação de aspectos de estrutura, processo e resultados da atenção à saúde indígena. Vale destacar que os modelos de relatório que o sistema gera, em algumas situações, apresentam-se como excessivos ou se revestem de dificuldade de interpretação para os usuários.

Uma característica do SIASI é sua ênfase no indivíduo, ou seja, toda a entrada dos dados é feita a partir do nome da pessoa no módulo demográfico, sendo esse o módulo chave do sistema.
Foi o primeiro a ser implantado e é por meio dele que é feita a entrada dos dados de cada indígena, bem como o registro dos óbitos e dos nascimentos. Os dois outros módulos implantados (imunização e morbidade) funcionam vinculados a esse módulo.

Conforme já apontado, inicialmente o SIASI foi concebido para funcionamento on-line, sendo alimentado a partir dos pólos base e de onde seria feita a transmissão instantânea dos dados para a sede do sistema, na FUNASA, em Brasília. As dificuldades operacionais enfrentadas, como a inexistência ou o mau funcionamento dos equipamentos e a precariedade da rede de internet, impossibilitaram seu funcionamento conforme o planejamento inicial. A partir de 2002, o sistema passou a funcionar da seguinte forma: a modalidade Web (com base de dados sediada em Brasília), que permite a consulta e a geração de informações pela internet; e o SIASI local, que permite a entrada de dados e a geração de relatórios locais e o envio das informações consolidadas para o SIASI Web (os lotes são enviados periodicamente do SIASI local para o nível nacional).

Até 2004, o SIASI havia passado por três versões. Na primeira (versão 1.0), constava apenas o já referido módulo demográfico. Na segunda

Tabela 1

Estruturação e funcionamento do Sistema de Informação da Atenção à Saúde Indígena (SIASI).

\begin{tabular}{|c|c|c|c|}
\hline Módulo & Finalidade & Principais variáveis & Implantação/Funcionamento \\
\hline Demográfico & $\begin{array}{l}\text { Informações demográficas/ } \\
\text { cadastro individual }\end{array}$ & $\begin{array}{l}\text { Parentesco, nascimento, } \\
\text { óbito, residência }\end{array}$ & $\begin{array}{l}\text { Implantado em 2000, com as } \\
\text { modalidades local e Web }\end{array}$ \\
\hline Morbidade & $\begin{array}{l}\text { Informações epidemiológicas } \\
\text { e de produtividade das unidades } \\
\text { e dos profissionais }\end{array}$ & $\begin{array}{l}\text { Recursos humanos, } \\
\text { procedimentos, diagnósticos, } \\
\text { exames laboratoriais }\end{array}$ & $\begin{array}{l}\text { Em fase de implantação na } \\
\text { modalidade local. } \\
\text { Funcionando na modalidade Web }\end{array}$ \\
\hline Imunização & $\begin{array}{l}\text { Informações de vacinas aplicadas } \\
\text { e planejamento de doses a aplicar }\end{array}$ & $\begin{array}{l}\text { Idade, vacinas aplicadas } \\
\text { e a aplicar }\end{array}$ & $\begin{array}{c}\text { Em fase de implantação na modalidade local. } \\
\text { Funcionando na modalidade Web }\end{array}$ \\
\hline Nutrição & $\begin{array}{l}\text { Informações sobre o estado } \\
\text { nutricional dos grupos de alto risco } \\
\text { (idosos, crianças, gestantes e nutrizes) }\end{array}$ & * & $\begin{array}{l}\text { Não implantado. } \\
\text { Para funcionamento na modalidade local }\end{array}$ \\
\hline Saúde bucal & $\begin{array}{l}\text { Informações sobre as ações } \\
\text { de saúde bucal }\end{array}$ & * & $\begin{array}{l}\text { Não implantado. } \\
\text { Para funcionamento na modalidade local }\end{array}$ \\
\hline $\begin{array}{l}\text { Acompanhamento } \\
\text { à gestação }\end{array}$ & Informações sobre o pré-natal & * & $\begin{array}{l}\text { Não implantado. } \\
\text { Para funcionamento na modalidade local }\end{array}$ \\
\hline Recursos humanos & $\begin{array}{l}\text { Informações de capacitação, } \\
\text { formação e quantitativo de pessoal }\end{array}$ & * & $\begin{array}{l}\text { Não implantado. } \\
\text { Para funcionamento na modalidade Web }\end{array}$ \\
\hline Infra-estrutura & Informações sobre infra-estrutura & * & $\begin{array}{l}\text { Não implantado. } \\
\text { Para funcionamento na modalidade Web }\end{array}$ \\
\hline
\end{tabular}

* Ainda não desenvolvido.

Fonte: Fundação Nacional de Saúde 24. 
(versão 2.0, de 2002), foi incluído o módulo de morbidade. Na terceira (versão 2.1.9, de 2004), foi introduzido o módulo de imunização.

O chamado "Documento Base" do SIASI 14 define o percurso dos dados e das informações no sistema, além de estabelecer as responsabilidades de cada instância. Segundo esse documento, nas aldeias seriam produzidos os dados que devem ser coletados em instrumentos padronizados a serem posteriormente encaminhados para os respectivos pólos bases. Em cada pólo, os dados deveriam ser digitados no SIASI local, na ocasião sendo emitidos relatórios e análises referentes às respectivas áreas de abrangência, providenciando-se o envio dos lotes via meio eletrônico (internet ou disquete) para a FUNASA em Brasília. No caso de pólos base que não tivessem capacidade operacional para essas atividades, o DSEI assumiria a responsabilidade de produção de relatórios e o envio dos dados. À FUNASA competiria coordenar o funcionamento do SIASI, gerenciando a base de dados nacional.

A Tabela 2 apresenta uma caracterização do SIASI local, explicitando sua utilidade, lógica de funcionamento e embasamento legal, além de tipos e fontes de dados e instrumentos de coleta.

\section{Potencialidades e limitações dos módulos do SIASI}

Quando comparado ao SINASC, algumas variáveis consideradas fundamentais para uma análise epidemiológica mais acurada dos nascimentos estão ausentes no SIASI, tais como o peso ao nascer e Apgar do recém-nascido, tipo de gravidez, número de consultas de pré-natal e número de filhos tidos anteriormente. Vale destacar que é possível que algumas dessas variáveis venham a ser incorporadas nos módulos do sistema ainda a serem implantados (como no de acompanhamento da gestação). Não obstante, face à proeminência do segmento infantil na população indígena e às altas taxas de fecundidade das mulheres em muitas etnias $7,10,15$, seria esperado que esse componente tivesse sido disponibilizado nas fases iniciais de implantação do sistema.

Dentre as variáveis do SIM que não constam no SIASI destacam-se: tipo de óbito, local de ocorrência do óbito e as variáveis relacionadas à condição do óbito. Assim como no caso dos nascimentos, a análise sobre a mortalidade deixa de contemplar aspectos importantes para o conhecimento e a avaliação da situação de saúde dos povos indígenas.

No SIASI, a inclusão dos dados referentes ao óbito é feita no módulo demográfico, estando disponíveis apenas as seguintes informações: data do óbito; se o óbito foi notificado ou não (entenda-se oportunamente); se a pessoa estava grávida e a causa da morte. Para a inclusão da causa do óbito, o próprio digitador seleciona o código com base na Classificação Internacional de Doenças - 10a Revisão 16 .

O módulo de morbidade do SIASI, desde sua implantação, apresentou-se bastante abrangente, contendo desde dados referentes a consultas realizadas, exames efetuados, referência e contra-referência, até os medicamentos administrados com seus respectivos custos. A mesma

Tabela 2

Principais características do Sistema de Informação da Atenção à Saúde Indígena (SIASI).

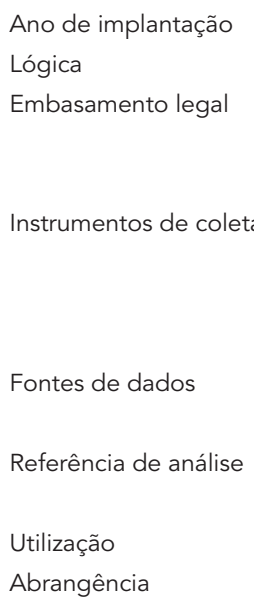

\section{0}

Epidemiológica e de serviços

Portaria no. 254/2002, que aprova a Política Nacional de

Atenção à Saúde dos Povos Indígenas 9.

Conferências Nacionais dos Povos Indígenas

Cadastro da família, formulário de consulta, prontuário, livro

do agente indígena de saúde (AIS), consolidado mensal das

atividades, formulário de referência e contra-referência

e relatório de imunizações

Aldeias, Casas de Saúde do Índio (CASAI) e unidades de

saúde públicas e privadas

Óbitos, nascimentos, morbidade, imunização, produção

de serviços

Vigilância, planejamento, avaliação e controle

População indígena 
tela de entrada possibilita registrar os tipos de atendimentos realizados, como consultas, procedimentos efetuados, acompanhamentos ao paciente, tratamentos realizados, assim como os dados relacionados à referência e contra-referência. Apesar da abrangência desse módulo, o mesmo apresenta limitações quando analisado na perspectiva da vigilância em saúde, chamando atenção para a falta de oportunidade em notificar e investigar as doenças de notificação compulsória.

Da forma como concebido, o módulo de imunização do SIASI permitiria acompanhar a situação vacinal de cada indivíduo, além de fornecer subsídios para o planejamento da administração das vacinas no DSEI. Está constituído pela inclusão dos seguintes dados: tipo de vacina aplicada, data, dose e lote. Permite ainda elaborar automaticamente todo o esquema vacinal obrigatório que cada indivíduo deve receber. Este módulo é, portanto, um bom instrumento para o planejamento das ações, desde que alimentado rotineiramente, bem como para a vigilância, pois permite identificar a população vacinada e a vacinar.

\section{Discussão}

No âmbito do desenvolvimento de sistemas de informação em saúde, Camargo Jr. \& Coeli 17 fazem uma distinção entre informação e informática, enfatizando que, na prática, predomina uma ênfase na última, restringindo as perspectivas de abordagem e as possibilidades de desenvolvimento da informação. A partir das análises conduzidas neste trabalho, percebe-se que, tal como sinalizado por esses autores, também no caso da concepção do SIASI houve uma ênfase no componente de informatização, resultando em relevância aquém da necessária em aspectos de informação de crucial importância.

Segundo Duarte et al. 18, o desenvolvimento tecnológico, aliado à ampliação da interface do setor saúde com a informática, facilitou o armazenamento e o processamento rápido dos dados. Enfatizam, ainda, que esse arranjo não foi necessariamente acompanhado pela ampliação da capacidade de análise e de interpretação dos dados pelos profissionais de saúde no nível local. Para esses autores, existem situações nas quais a discussão sobre os sistemas informatizados se sobrepõe às discussões da vigilância, como se essa não pudesse existir sem os sistemas informatizados. No caso do SIASI, vê-se uma situação na qual a coleta, o armazenamento e o processamento dos dados, apesar dos recursos de informatização disponíveis e dos problemas apontados, não vieram associados a uma ampliação, na escala necessária, da capacidade de análise e de emprego das informações em saúde para fins da execução e avaliação das ações.

Uma das deficiências na concepção do SIASI diz respeito às variáveis a serem utilizadas nos instrumentos de coleta. Deixaram de ser incluídas variáveis importantes para a construção de um perfil epidemiológico, ao mesmo tempo em que foram inseridas outras de menor relevância. Esse fato ocasionou um número excessivo de dados, sem o conhecimento e reconhecimento da sua importância pelos profissionais usuários, levando a um desinteresse ou ao não preenchimento desses formulários na rotina da atenção à saúde indígena.

Recuperando-se a trajetória de criação do SIASI a partir dos documentos analisados, observou-se que as discussões ficaram restritas a um número reduzido de técnicos do nível central, com pouca participação de profissionais da área da vigilância. Vale destacar que, na época de criação do SIASI, o Centro Nacional de Epidemiologia (CENEPI), atual Secretaria de Vigilância em Saúde (SVS), ligado ao Ministério da Saúde e responsável pela normatização das ações de vigilância e gerência da maioria dos sistemas de informação em saúde, era vinculado à FUNASA. Não foram localizadas, nos materiais analisados, evidências de sua participação sistemática na concepção do sistema.

É importante lembrar também que a qualidade de um sistema de informação depende muito da qualidade dos dados coletados, incluindo a regularidade e o correto preenchimento dos instrumentos. O fato de os instrumentos utilizados pelo SIASI serem preenchidos por uma diversidade de profissionais, desde o agente indígena de saúde até o médico da equipe, aumenta as possibilidades de inconsistências e também de incorreções. Além da ausência de capacitação específica para lidar com os instrumentos de coleta, os profissionais de saúde defrontam-se com uma rotina de preencher um excessivo número de instrumentos de coleta. Aliado a isso, existe a alta rotatividade de recursos humanos que trabalham com a saúde indígena, com deficiência de treinamentos específicos e adequados, bem como de supervisão e acompanhamento sistemático desses profissionais 3,19 .

A comunicação do SIASI com os diversos sistemas de informação do Ministério da Saúde, apesar de fazer parte da sua concepção, ainda está para se efetivar. Em decorrência, surgem inconsistências importantes. Por exemplo, apesar de o campo raça/cor estar presente na declaração de óbito desde 1996, o número de óbitos da população indígena é, pelo menos, três vezes menor no SIM quando comparado ao SIASI. Do- 
cumentos internos da FUNASA já demonstram como prioridade a necessidade de articulação intra-institucional visando compatibilizar os sistemas de informação, tornando o SIASI mais afim aos demais do Ministério da Saúde.

Diferente de outros sistemas de informação no país (como o SIM, SINASC, entre outros), nos quais o acesso às informações é facultado a qualquer cidadão, o SIASI não disponibiliza as informações produzidas para acesso ao público, mesmo se agregadas. Apenas os gestores da saúde indígena e os profissionais que atuam no órgão têm acesso permitido, por meio de uma senha disponibilizada pela FUNASA. Isso contraria um dos propósitos de sua criação, que é o de facilitar a participação comunitária no acompanhamento das ações de saúde.

Existem diversos outros aspectos extremamente frágeis na concepção e operacionalização do SIASI. Em particular, a normatização da rotina e do fluxo para a notificação dos casos de doenças de notificação compulsória, bem como a sistematização do uso das fichas de notificação e investigação dessas doenças, ainda não estão totalmente implantadas. Há também a subnotificação da morbidade em geral, decorrente da descontinuidade da assistência nas aldeias, tornando-se praticamente impossível resgatar essa informação nos períodos de ausência das equipes das áreas indígenas. A não permanência das equipes em área também ocasiona uma demora no envio dos dados para as demais instâncias.

Essas limitações impossibilitam que o SIASI funcione, de fato, como um instrumento para a vigilância em saúde. Segundo Hammann \& Laguardia 20, existe consenso acerca da necessidade de um processo institucionalizado de notificação acoplado a um sistema de informação ágil, constituindo pilares das ações de vigilância. O que se percebe, a partir da análise dos documentos norteadores do SIASI, é que a finalidade primordial do módulo de morbidade parece não ser a vigilância, mas sim monitorar a resolubilidade e a qualidade da assistência ao paciente.

Em relação aos nascimentos e óbitos, nota-se, no SIASI, a ausência de variáveis importantes (a exemplo das coletadas pelo SIM e pelo SINASC), o que inviabiliza ou dificulta a utilização do sistema para a vigilância em saúde e a monitorização, essa última entendida no seu aspecto mais abrangente. A ausência de dados importantes do recém-nascido, da mãe e da gestação impossibilita que seja efetuada uma vigilância dos recém-nascidos de risco, algo fundamental, dada a grande magnitude da mortalidade infantil na população indígena 5,6,7.

É importante frisar que muitas das limitações do SIASI enquadram-se nas críticas sobre os sis- temas de informação referidas no III Plano Diretor para o Desenvolvimento da Epidemiologia no Brasil para o período 2000-200421. Constituem deficiências também identificadas, ainda que em menor magnitude, no Sistema de Informação de Agravos de Notificação (SINAN), conforme apontam Laguardia et al. 22, e também em outros sistemas de informações de interesse epidemiológico existentes no país.

Apesar das inúmeras limitações identificadas, tanto na concepção quanto na operacionalização, há que se reconhecer que o SIASI apresenta potencialidades. Dentre elas, destaca-se a integralidade, ou seja, o fato de se propor a agregar, em um único sistema, uma multiplicidade de informações necessárias para o conhecimento da realidade de saúde dos povos indígenas. Outro aspecto positivo refere-se às possibilidades de análise, permitindo a desagregação de dados no nível de aldeias e etnias, e até mesmo de unidade familiar e indivíduo. O sistema disponibiliza dados sobre a composição das famílias, permitindo realizar a transferência de dados entre aldeias, ou até mesmo entre DSEI.

Ainda quanto às potencialidades, o SIASI é um sistema relativamente simples, de fácil manuseio, podendo ser operacionalizado e analisado nas instâncias locais. Isso desde que os recursos humanos existentes sejam capacitados para desenvolver essas análises e que existam organização dos serviços e uma capacidade mínima instalada. Assim, se o modelo de gestão e organização de serviços preconizados para os DSEI vier a ser cumprido de fato, permitirá um monitoramento das condições de saúde nas comunidades indígenas, desde que feitos ajustes estruturais no sistema. O modelo, pautado na atenção primária, com equipes permanentemente em áreas e subsidiado com um sistema de informação adequado, diminuiria a subnotificação de nascimentos, doenças e óbitos. Ampliaria também as possibilidades de intervenções oportunas, ou seja, a identificação e o controle dos problemas identificados em cada aldeia, contribuindo para minimizar a grave situação de saúde dos povos indígenas no país $4,5,6$.

Por fim, há que se ressaltar que a existência de um sistema específico para os povos indígenas justifica-se ao se considerar seu reduzido tamanho populacional, aliado às suas especificidades socioculturais. Não obstante, conforme ressaltado neste trabalho, há a urgente necessidade de repensar vários aspectos da estrutura e funcionamento do SIASI, de modo a ampliar suas potencialidades para a vigilância em saúde. 


\section{Resumo}

O Sistema de Informação da Atenção à Saúde Indígena (SIASI) foi criado no âmbito do Subsistema de Atenção à Saúde Indígena, a partir de 1999. Visou melhorar a disponibilidade e o acesso às informações sobre saúde, fundamentais para o planejamento, operação e avaliação das ações. Este trabalho analisa o SIASI, contemplando aspectos relativos à sua criação, implantação, funcionamento, potencialidades e limitações. Procedeu-se levantamento documental no Departamento de Saúde Indígena da Fundação Nacional de Saúde, complementado com análises comparativas com outros sistemas de informação em saúde. O SIASI se estrutura em oito módulos, três dos quais em operação. As principais limitações do SIASI referem-se aos instrumentos de coleta, à capacitação dos recursos humanos, à ausência de interface com os demais sistemas nacionais de informação em saúde, à dificuldade de acesso às informações e a não utilização das informações para o planejamento das ações. Entre as potencialidades, destacam-se a simplicidade do sistema, sua integralidade e as possibilidades de desagregações. Frisa-se a importância de um sistema de informação em saúde específico para os povos indígenas em decorrência de suas especificidades socioculturais e demográficas.

Sistemas de Informação; Política de Saúde; Índios SulAmericanos

\section{Colaboradores}

M. C. Sousa coletou e analisou os dados da pesquisa que serviram de base para este artigo. J. H. G. Scatena e R. V. Santos participaram da concepção do projeto que originou o artigo. Todos os autores participaram da elaboração e revisão deste texto.

\section{Agradecimentos}

À Fundação Nacional de Saúde (FUNASA), pelo apoio logístico para a realização da pesquisa. Aos técnicos do Departamento de Saúde Indígena (DESAI), especialmente Carlos Coloma e Edgard Magalhães, que possibilitaram e facilitaram o levantamento documental sobre o SIASI e ofereceram importantes contribuições verbais. Também a Paulo C. Sabroza (Escola Nacional de Saúde Pública Sergio Arouca, Fundação Oswaldo Cruz/Rio de Janeiro) e a Paulo S. Camargo (FUNASA/Rio de Janeiro) pela leitura crítica e comentários.

\section{Referências}

1. Branco MAF. Informação em saúde como elemento estratégico para a gestão. In: Ministério da Saúde, organizador. Gestão municipal de saúde: textos básicos. Brasília: Ministério da Saúde; 2001. p. 163-9.

2. Moraes IHS. Política, tecnologia e informação em saúde: a utopia da emancipação. Salvador: Casa da Qualidade; 2002.

3. Rede Interagencial de Informações para a Saúde. Indicadores básicos de saúde no Brasil: conceitos e aplicações. Brasília: Organização Pan-Americana da Saúde; 2002.

4. Coimbra Jr. CEA, Santos RV. Minorias e desigualdades: algumas teias de inter-relações, com ênfase nos povos indígenas no Brasil. Ciênc Saúde Coletiva $2000 ; 5: 125-32$.
5. Garnelo L, Macedo G, Brandão LC. Os povos indígenas e as políticas de saúde no Brasil. Brasília: Organização Pan-Americana da Saúde/Ministério da Saúde; 2003.

6. Santos RV, Coimbra Jr. CEA. Cenários e tendências da saúde e da epidemiologia dos povos indígenas no Brasil. In: Santos RV, Coimbra Jr. CEA, Escobar $\mathrm{AL}$, organizadores. Epidemiologia e saúde dos povos indígenas no Brasil. Rio de Janeiro: Editora Fiocruz/ABRASCO; 2003. p. 13-48.

7. Cardoso AM, Santos RV, Coimbra Jr. CEA. Mortalidade infantil segundo raça/cor no Brasil: o que dizem os sistemas nacionais de informação? Cad Saúde Pública 2005; 21:1602-8. 
8. Montenegro RA, Stephens C. Indigenous health in Latin America and the Caribbean. Lancet 2006; 367:1859-69.

9. Fundação Nacional de Saúde. Política nacional de atenção à saúde dos povos indígenas. 2a Ed. Brasília: Fundação Nacional de Saúde/Ministério da Saúde; 2002.

10. Pagliaro H, Azevedo MM, Santos RV, organizadores. Demografia dos povos indígenas no Brasil. Rio de Janeiro: Editora Fiocruz/Associação Brasileira de Estudos Populacionais; 2005.

11. Brasil. Lei no. 9.836 de 23 de setembro de 1999. Acrescenta dispositivos à Lei 8.080 de 19/09/1990, instituindo o subsistema de atenção à saúde indígena. Diário Oficial da União 1999; 24 set.

12. Ministério da Saúde. Relatório final da Conferência Nacional de Proteção à Saúde do Índio. Tema específico da 8a Conferência Nacional de Saúde. Brasília: Ministério da Saúde; 1986.

13. Ministério da Saúde. Relatório final da III Conferência Nacional de Saúde dos Povos Indígenas. Luziânia/Brasília: Ministério da Saúde; 2001.

14. Coloma C. Documento base: sistema de informações para a atenção à saúde do índio, Brasília: Ministério da Saúde/Fundação Nacional de Saúde; 2002.

15. Instituto Brasileiro de Geografia e Estatística. Tendências demográficas: uma análise dos indígenas com base nos resultados da amostra dos censos demográficos 1991 e 2000. Rio de Janeiro: Instituto Brasileiro de Geografia e Estatística; 2005.

16. Organização Mundial da Saúde. Classificação estatística internacional de doenças e de problemas relacionados à saúde, 10a revisão. São Paulo: Centro Colaborador da OMS para a Classificação de Doenças em Português; 1995.
17. Camargo Jr. K, Coeli CM. Políticas de informação em saúde. In: Escola Politécnica de Saúde Joaquim Venâncio, organizadora. Textos de apoio em políticas de saúde. Rio de Janeiro: Editora Fiocruz; 2005. p. 143-56.

18. Duarte EC, Schneider MC, Paes-Souza R, Ramalho WM, Sardinha LMV, Silva Junior JB, et al. Epidemiologia das desigualdades em saúde no Brasil: um estudo exploratório. Brasília: Organização Pan-Americana da Saúde; 2002.

19. Chaves MBG, Cardoso AM, Almeida C. Implementação da política de saúde indígena no Pólo-base Angra dos Reis, Rio de Janeiro, Brasil: entraves e perspectivas. Cad Saúde Pública 2006; 22:295-305.

20. Hammann EM, Laguardia J. Reflexões sobre a vigilância epidemiológica: mais além da notificação compulsória. Inf Epidemiol SUS 2000; 9:211-9.

21. ABRASCO. III plano diretor para o desenvolvimento da epidemiologia no Brasil 2000-2004. Rio de Janeiro: ABRASCO; 2000.

22. Laguardia J, Domingues CMA, Carvalho C, Lauerman CR, Macário E, Glatt R. Sistema de Informação de Agravos de Notificação (SINAN): desafios no desenvolvimento de um sistema de informação em saúde. Inf Epidemiol SUS 2004; 13:135-47.

23. Ministério da Saúde. Portaria no. 70, de 20 de janeiro de 2004. Diário Oficial da União 2004; 22 jan.

24. Fundação Nacional de Saúde. Documento sobre o Sistema de Informação da Atenção à Saúde Indígena. Brasília: Fundação Nacional de Saúde/Ministério da Saúde; 2004.

\section{Recebido em 13/Fev/2006}

Versão final reapresentada em 13/Nov/2006

Aprovado em 14/Nov/2006 